\title{
Direct Position Determination by Double Fixed Station Based on Delay and AOA
}

\author{
Xiangyu Liu*, Qing He and Zhengbo Sun \\ National Key Laboratory of Science and Technology on Blind Signal Processing, Chengdu, Sichuan, 86-610041, China \\ ${ }^{*}$ Corresponding author
}

\begin{abstract}
To improve location accuracy, a single-step localization algorithm by double fixed station, using the thought of "signal to position" is proposed to solve the problem of two-step conventional method's information loss, since two-step conventional method divides in estimating intermediate parameter and geolocation. First, the observed signal model is analyzed and problem's mathematical model is generalized. Next, the cost function is formulated based on maximum likelihood estimator (MLE) and simplified as the maximal eigenvalue of hermite matrix. Then, the geographical location maps in twodimensional sector-grid based on angel of arrival (AOA), afterward, the algorithm process is introduced. Finally, simulation results demonstrated that the proposed DPD algorithm outperforms the two-step conventional algorithm in location accuracy, and when signal to noise ratio (SNR) of the

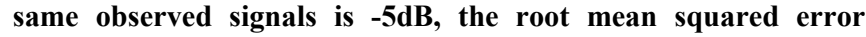
(RMSE) of proposed algorithm reduce the errors of $47 \%$ in typical scene.
\end{abstract}

Keywords-passive localization; direct position determination (DPD); maximum likelihood estimator (MLE); passive localization; time delay; angel of arrival (AOA)

\section{INTRODUCTION}

Passive localization has been paid much more attention for its outstanding performance in self-hiding, far-distance detection and extensive applicability because it dosen't transmit signals but receives signals [1]. As for narrowband signals, passive localization of combined time delay and AOA is a relatively good option. On the one hand, accuracy of estimating time difference of arrival (TDOA) parameter is very low and the information of AOA can reduce searching scope and improve localization precision. On the other hand, the proposed algorithm which can reduce the number of observed stations, has good application prospects.

Passive localization can be divided into the two-step conventional methods and the single-step methods (direct position determination, DPD), according to algorithm steps. The two-step conventional methods measure time difference of arrivals (TDOA) and angel of arrival (AOA) in the first step, and use these measurements for geographical localization in the second step, while the direct position determination methods which are focused in this paper for higher location accuracy, do not need intermediate parameter and can directly get target location through observed signals. For DPD method, many scholars have done research on it. Israeli scientist Weiss proposed the direct position determination concept for the first time in 2004 [2] [3]. Oispuu M extended direct position determination algorithm to a moving antenna array in the case of a time-varying number of emitting sources in 2010 [4] [5]. The team of Steven Kay proposed a weighted least squares type position fixing technique for only TDOA direct position determination algorithm in 2013 [6] [7]. Zhiyin Huang in the information engineering university introduced multi-array direct position determination algorithm and considered unknown non-uniform noise in time domain and frequency domain [8].

However, the above existing direct position determination methods only pay attention on collaborative localization algorithm of homogeneous sensors. In this paper, we shall propose a novel direct position determination algorithm of heterogeneous sensors based on delay and AOA to overcome the shortcoming of the two-step conventional methods.

\section{PROBLEM Formulation}

Consider a scenario which has several separated receivers and a stationary transmitter which figure 1 shows. The main station has an array antenna and a time difference antenna, while each assistant station only have a time difference antenna, and can send observed signals to the main station through signal repeaters.

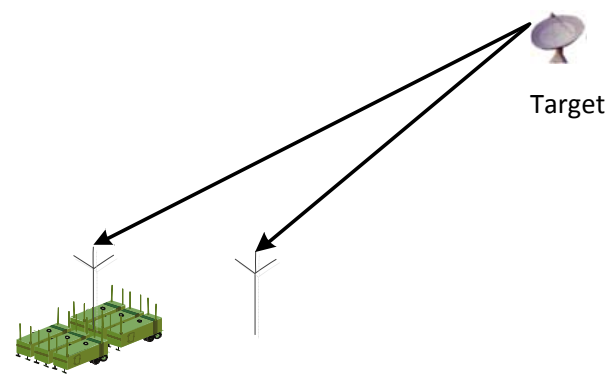

Main station Assistant station

FIGURE I LOCALIZATION SCENE

Assume that the receiving stations are strictly synchronized. Considering that the discrete signal which the $m$ th receiver sampled is $r_{m}(n)$ from time difference antenna, while the discrete signal which the main station sampled is $\tilde{r}(n)$. Then the receivers' output can be modeled as 


$$
\begin{cases}r_{m}(n)=b_{m} s\left(n-D_{m}\right)+w_{m}(n), & n=0,1, \cdots, N-1 \\ \tilde{r}(n)=a\left(\mathbf{p}_{t}\right) \tilde{s}(n)+\tilde{w}(n), & n=0,1, \cdots, \tilde{N}-1\end{cases}
$$

where $s(n)$ is the complex envelope of the transmitter, $w_{m}(n)$ is a wide-sense stationary, white, zero mean, complex, Gaussian noise, $b_{m}$ is an unknown channel attenuation factor, $D_{m}=\frac{1}{c}\left\|\mathbf{p}_{t}-\mathbf{p}_{m}\right\|$ is time difference between transmitter to $m$ th receiver, $\mathbf{p}_{t}$ is the geographical position of transmitter. $\mathbf{p}_{m}$ is the geographical position of $m$ th receiver, $N$ is sampling number, $M$ is the number of receivers, $a\left(\mathbf{p}_{t}\right)$ is the array response to a signal transmitted from position $\mathbf{p}_{t} \cdot \tilde{w}_{m}(n)$ is a wide-sense stationary, white, zero mean, complex, Gaussian noise from array antenna.

Transform the signal to the frequency domain as matrix form

$$
\left\{\begin{array}{l}
\mathbf{r}_{m}=b_{m} \boldsymbol{\Phi}_{m} \mathbf{s}+\mathbf{w}_{m}, \quad m=1,2, \cdots, M \\
\tilde{\mathbf{r}}=A\left(\mathbf{p}_{t}\right) \tilde{\mathbf{s}}+\tilde{\mathbf{w}}
\end{array}\right.
$$

where we defined

$$
\begin{aligned}
& \mathbf{r}_{m} \square\left[r_{m}\left(f_{0}\right), \cdots, r_{m}\left(f_{K-1}\right)\right]^{T} \\
& \mathbf{s} \square\left[s\left(f_{0}\right), \cdots, s\left(f_{K-1}\right)\right]^{T} \\
& \mathbf{w}_{m} \square\left[w_{m}\left(f_{0}\right), \cdots, w_{m}\left(f_{K-1}\right)\right]^{T} \\
& \boldsymbol{\Phi}_{m} \square \operatorname{diag}\left\{\left[e^{-j 2 \pi D_{m} T_{s} f_{0}}, \cdots, e^{-j 2 \pi D_{m} T_{s} f_{K-1}}\right]\right\} \\
& \tilde{\mathbf{s}} \square\left[\tilde{s}\left(f_{0}\right), \cdots, \tilde{s}\left(f_{K-1}\right)\right]^{T} \\
& \tilde{\mathbf{w}} \square\left[\tilde{w}\left(f_{0}\right), \cdots, \tilde{w}\left(f_{K-1}\right)\right]^{T}
\end{aligned}
$$

The information of target $\mathbf{p}_{t}$ is included in time delay $\boldsymbol{\Phi}_{m}$ and the array response $A\left(\mathbf{p}_{t}\right)$ from array antenna.

Thus, it is now clear that the localization problem can be modeled that given observed signals $\mathbf{r}_{m}=b_{m} \boldsymbol{\Phi}_{m} \mathbf{s}+\mathbf{w}_{m}$ and $\tilde{\mathbf{r}}=A\left(\mathbf{p}_{t}\right) \tilde{\mathbf{s}}+\tilde{\mathbf{w}}$ and receivers' position $\mathbf{p}_{m}$, estimate the position of transmitter $\mathbf{p}_{t}$.

\section{The Two-step Conventional Algorithm}

In the first step, time difference of arrivals (TDOA, $\Delta t$ ) shall be estimated though cross-correlation method, using the main station and the assistant station observed signals, while angel of arrival (AOA, $\theta$ ) can be estimated though multiple signal classification (MUSIC) method, using the main station observed signals. Geometrically, the intersection of hyperbola via $\Delta t$ and ray via $\theta$ is the target location.

Consider double station and target's rectangular coordinates are $O_{1}\left(x_{1}, y_{1}\right), O_{2}\left(x_{2}, y_{2}\right), T(x, y)$. We can obtain equations

$$
\left\{\begin{array}{l}
\Delta r=\sqrt{\left(x-x_{2}\right)^{2}+\left(y-y_{2}\right)^{2}}-\sqrt{\left(x-x_{1}\right)^{2}+\left(y-y_{1}\right)^{2}}=c \square \Delta t \\
\tan \theta=\frac{y-y_{1}}{x-x_{1}}
\end{array}\right.
$$

Expressing (4) in matrix form, we have

$$
\left[\begin{array}{cc}
x_{0}-x_{1} & y_{0}-y_{1} \\
x_{0}-x_{2} & y_{0}-y_{2}
\end{array}\right]\left[\begin{array}{l}
x \\
y
\end{array}\right]=\left[\begin{array}{l}
k+r_{1} \cdot \Delta r \\
\tan \theta \llbracket x_{1}-y_{1}
\end{array}\right]
$$

where $\quad r_{1}=\sqrt{\left(x-x_{1}\right)^{2}+\left(y-y_{1}\right)^{2}} \quad$ and

$k=\frac{1}{2}\left[\left(x_{1}^{2}+y_{1}^{2}\right)-\left(x_{2}^{2}+y_{2}^{2}\right)+\Delta r^{2}\right]$,

$$
\left[\begin{array}{l}
\hat{x} \\
\hat{y}
\end{array}\right]=\left[\begin{array}{l}
m_{1}+n_{1} \cdot r_{1} \\
m_{2}+n_{2} \cdot r_{1}
\end{array}\right]
$$

Substituting (6) into $r_{1}=\sqrt{\left(x-x_{1}\right)^{2}+\left(y-y_{1}\right)^{2}}$, we get

$$
s_{1} r_{1}^{2}+s_{2} r_{1}+s_{3}=0
$$

Solve the quadratic equation (7), we get $r_{1}$, then substitute (6), we arrive target coordinate $T(x, y)$. As algorithm proposed above, double receivers are able to identify the target location, which shall be applied to narrowband signals and few stations' scene.

\section{Direct Position DeterminAtion Algorithm BASED ON DELAY AND AOA}

\section{A. The DPD Estimator}

In this section, we shall formulate and simplify the cost function using maximum likelihood estimator (MLE). Assume observed noise is wide-sense stationary, white, zero mean, then the probability density function of noise can be expressed as

$$
f\left(\mathbf{w}_{m}\right)=\frac{1}{\left(\pi \sigma^{2}\right)^{m}} \exp \left(-\frac{1}{\sigma^{2}} \mathbf{w}_{m}{ }^{H} \mathbf{w}_{m}\right)=\frac{1}{\left(\pi \sigma^{2}\right)^{m}} \exp \left(-\frac{1}{\sigma^{2}}\left\|\mathbf{w}_{m}\right\|\right)
$$
is

After taking the logarithm, the likelihood function of noise

$$
L\left(\mathbf{w}_{m}\right)=\log f\left(\mathbf{w}_{m}\right)=-m \cdot \log \left(\pi \sigma^{2}\right)-\frac{1}{\sigma^{2}}\left\|\tilde{\mathbf{r}}_{m}-\mathbf{A} b_{m}\right\|^{2}
$$

Then, maximum likelihood solution is 


$$
\begin{aligned}
\hat{\mathbf{p}}_{\mathrm{ML}} & =\underset{\mathbf{p}_{t}}{\arg \max }\left\{-m \cdot \log \left(\pi \sigma^{2}\right)-\frac{1}{\sigma^{2}}\left\|\tilde{\mathbf{r}}_{m}-\mathbf{A} b_{m}\right\|^{2}\right\} \\
& =\underset{\mathbf{p}_{t}}{\arg \min }\left\|\tilde{\mathbf{r}}_{m}-\mathbf{A} b_{m}\right\|^{2} \\
& =\underset{\mathbf{p}_{t}}{\arg \min } F_{1}\left(\mathbf{p}_{t}\right)
\end{aligned}
$$

where $F_{1}\left(\mathbf{p}_{t}\right)=\left\|\tilde{\mathbf{r}}_{m}-\mathbf{A} b_{m}\right\|^{2}$ is the cost function of MLE, $\mathbf{p}_{t}$ is the locate vector of target, $\mathbf{A}=\boldsymbol{\Phi}_{m} \tilde{\mathbf{s}}$. In the above section, we formulate the cost function, using maximum likelihood estimator, but we can't calculate it directly, since the complex envelope of the transmitter $s(n)$ and channel attenuation factor $b_{m}$ is unknown. In the following section, we shall simplify the cost function to calculate it.

When matrix $\mathbf{A}^{H} \mathbf{A}$ is invertible, transforming (10), we can get

$$
b_{m}=\left(\tilde{\mathbf{s}}^{H} \boldsymbol{\Phi}_{m}{ }^{H} \boldsymbol{\Phi}_{m} \tilde{\mathbf{s}}\right)^{-1}\left(\boldsymbol{\Phi}_{m} \tilde{\mathbf{s}}\right)^{H} \tilde{\mathbf{r}}_{m}=\frac{1}{\left\|\boldsymbol{\Phi}_{m}\right\|^{2}\|\tilde{\mathbf{s}}\|^{2}}\left(\boldsymbol{\Phi}_{m} \tilde{\mathbf{s}}\right)^{H} \tilde{\mathbf{r}}_{m}
$$

Supposing $\|\tilde{\mathbf{s}}\|^{2}=1,\left\|\mathbf{\Phi}_{m}\right\|^{2}=\mathbf{I}$ and substituting (11) into (10), gets

$$
\begin{aligned}
F_{2}\left(\mathbf{p}_{t}\right) & =\sum_{m=1}^{M}\left\|\tilde{\mathbf{r}}_{m}-\boldsymbol{\Phi}_{m} \tilde{\mathbf{s}}\left(\boldsymbol{\Phi}_{m} \tilde{\mathbf{s}}\right)^{H} \tilde{\mathbf{r}}_{m}\right\|^{2} \\
& =\sum_{m=1}^{M}\left\{\tilde{\mathbf{r}}_{m}{ }^{H} \tilde{\mathbf{r}}_{m}-\tilde{\mathbf{r}}_{m}{ }^{H} \boldsymbol{\Phi}_{m} \tilde{\mathbf{s}}\left(\boldsymbol{\Phi}_{m} \tilde{\mathbf{s}}\right)^{H} \tilde{\mathbf{r}}_{m}\right\} \\
& =\sum_{m=1}^{M}\left\|\tilde{\mathbf{r}}_{m}\right\|^{2}-\sum_{m=1}^{M}\left|\tilde{\mathbf{r}}_{m}{ }^{H} \boldsymbol{\Phi}_{m} \tilde{\mathbf{s}}\right|^{2}
\end{aligned}
$$

Minimizing (12) is equal to maximizing $F_{3}(\mathbf{p})$

$$
F_{3}\left(\mathbf{p}_{t}\right)=\sum_{m=1}^{M}\left|\tilde{\mathbf{r}}_{m}{ }^{H} \boldsymbol{\Phi}_{m} \tilde{\mathbf{s}}\right|^{2}=\sum_{m=1}^{M} \tilde{\mathbf{s}}^{H} \boldsymbol{\Phi}_{m}{ }^{H} \tilde{\mathbf{r}}_{m} \tilde{\mathbf{r}}_{m}{ }^{H} \boldsymbol{\Phi}_{m} \tilde{\mathbf{s}}=\tilde{\mathbf{s}}^{H} \mathbf{Q} \tilde{\mathbf{s}}
$$

where $\mathbf{Q}=\mathbf{V} \mathbf{V}^{H} \quad \mathbf{V}=\left[\boldsymbol{\Phi}_{1}{ }^{H} \tilde{\mathbf{r}}_{1}, \cdots, \boldsymbol{\Phi}_{M}{ }^{H} \tilde{\mathbf{r}}_{M}\right]$.

To reduce the amount of computing, maximizing $F_{3}(\mathbf{p})$ is equal to $F_{4}(\mathbf{p})$

$$
F_{4}(\mathbf{p})=\lambda_{\text {max }}\{\mathbf{Q}\}=\lambda_{\text {max }}\{\tilde{\mathbf{Q}}\}
$$

where $\tilde{\mathbf{Q}} \square \mathbf{V}^{H} \mathbf{V}$. Matrix $\tilde{\mathbf{Q}}$ is $M \times M$ dimension where $M$ is the number of receivers, while Matrix $\mathbf{Q}$ is $N \times N$ dimension where $N$ is the number of the sampling points and $M \square N$.

\section{B. Sector Grid Division}

In this section, we reduce searching scope based on angel of arrival and error range in the direct position determination information field which established in the A section. Assume that target's altitude is known, otherwise, add another dimension to search.

The angel $\theta$ from the grid point to main station as $\mathrm{X}$ axes and distance $r$ from the gridding point to main station as $\mathrm{Y}$ axes represent the sector area. Thus, section range $D_{X}$ is

$$
D_{X}=\left[\theta_{\min }, \theta_{\text {max }}\right] \otimes\left[r_{\min }, r_{\max }\right]
$$

Assume that the step length $\Delta \theta$ and $\Delta r$ divide sector area with the number of $N_{x} \times N_{y}$.

$$
N_{x}=\left\lfloor\frac{\theta_{\max }-\theta_{\min }}{\square \theta}\right\rfloor, N_{y}=\left\lfloor\frac{r_{\max }-r_{\min }}{\square r}\right\rfloor
$$

where $\lfloor\bullet\rfloor$ is round toward negative infinity. Thus, every gridding point represent $\mathbf{p}_{n_{x}, n_{y}}=\left(\theta_{n_{x}}, r_{n_{y}}\right)$

$$
\begin{array}{ll}
\theta_{n_{x}}=\theta_{\min }+n_{x} \Delta \theta, & r_{n_{y}}=r_{\min }+n_{y} \Delta r \\
n_{x}=0,1, \cdots, N_{x}, & n_{y}=0,1, \cdots, N_{y}
\end{array}
$$

\section{Algorithm Process}

After analyzing theory above, the direct position determination algorithm by double fixed station based on delay and AOA is as follows:

Step1: Set Monte Carlo simulation times, and Initialize receiving station's position $\mathbf{p}_{m}$

Step2: Load observed signals and do FFT transform, get $\mathbf{r}_{m}$

Step3: Determine the searching sector area $\mathbf{p}_{n_{x}, n_{y}}=\left(\theta_{n_{x}}, r_{n_{y}}\right)$ and grid of location $\mathbf{p}_{1}, \mathbf{p}_{2}, \cdots, \mathbf{p}_{N_{x} \times N_{y}}$, according to AOA

Step4: Calculate the time delay $D_{m}$ and $\boldsymbol{\Phi}_{m}, \mathbf{V}, \tilde{\mathbf{Q}}$

Step5: Evaluate the simplified cost function $F_{4}\left(\mathbf{p}_{i}\right)$

Step6: Find in which $\mathbf{p}_{i}, F_{4}(\mathbf{p})$ is the largest, thus, this grid point location is the estimated position.

\section{Step7: Calculate localization RMSE}

As algorithm process is proposed above, time is Monte Carlo simulation times, $\mathbf{p}_{n_{x}, n_{y}}=\left(\theta_{n_{x}}, r_{n_{y}}\right)$ is sector grid division, $F_{4}\left(\mathbf{p}_{i}\right)$ is formula (14), $N_{x} \times N_{y}$ is the sum number of grid points, $\tilde{\mathbf{Q}}$ is a $M \times M$ dimension Hermite matrix which is mentioned in (13). In addition, when targets is more than one, 
find in which $\mathbf{p}_{i}$, the direct position determination information field has apices, thus the number of apices is multi-target's number.

\section{NUMERICAL EXAMPLES}

In this section, we provide a numerical example of the proposed algorithm to examine the performance of the direct position determination by double fixed station based on delay and AOA. We simulate $3 \mathrm{kHz}$ narrowband signals and double station whose parameter is in table I.

\section{TABLE I. SIMILATION PARAMETER}

\begin{tabular}{|c|c|}
\hline Parameter Type & Parameter Value \\
\hline Signal Bandwidth & $3 \mathrm{kHz}$ \\
\hline Sampling & $15 \mathrm{kHz}$ \\
\hline Signal Time & $1 \mathrm{~s}$ \\
\hline Center frequency & $3 \mathrm{kHz}$ \\
\hline Symbol Number & 3000 \\
\hline Sector Angel Range & $0.1^{\circ}$ \\
\hline Sector Grid Point Number & $41^{*} 201$ \\
\hline SNR Range & $-20 \mathrm{~dB}$ to $25 \mathrm{~dB}$ \\
\hline Monte Carlo Times & 100 \\
\hline Main Station Coordinate & $(-35 \mathrm{~km},-35 \mathrm{~km})$ \\
\hline Assistant Station Coordinate & $(-15 \mathrm{~km},-35 \mathrm{~km})$ \\
\hline Target Coordinate & $(0 \mathrm{~km}, 0 \mathrm{~km})$ \\
\hline
\end{tabular}

To evaluate algorithm capability, root mean squared error ( RMSE $=\sqrt{\frac{1}{N} \sum_{\mathrm{i}=1}^{N}\left(\tilde{\mathbf{x}}_{i}-\mathbf{x}\right)\left(\tilde{\mathbf{x}}_{i}-\mathbf{x}\right)^{T}}$ ) which is inaccuracy standard is used to calculate the difference of the real values $\mathbf{x}$ and the measuring values $\mathbf{x}_{i}$.

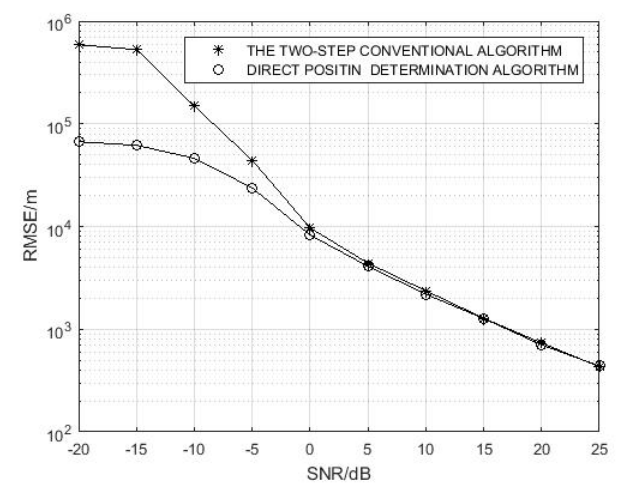

FIGURE II RMSE OF THE DPD ALGORITHM AND THE TWO-STEP CONVENTIONAL ALGORITHM

Figure II shows the root mean squared error as a function of SNR, in which the nether round line represents the proposed DPD algorithm and the upper star line represents the two-step conventional algorithm and $\mathrm{Y}$ axes displays logarithmic coordinate. When SNR varies from $0 \mathrm{~dB}$ to $25 \mathrm{~dB}$ at high level, the performance difference between two algorithms becomes smaller. When SNR varies from $-20 \mathrm{~dB}$ to $0 \mathrm{~dB}$ at low level, RMSE of the proposed algorithm is less than the conventional algorithm. For example, RMSE of the proposed algorithm is $23 \mathrm{~km}$, while RMSE of the conventional algorithm is $44 \mathrm{~km}$, which is reduced $47 \%$. Thus, the proposed algorithm performs better in accuracy than the conventional algorithm.

\section{CONCLUSION}

In this paper, a direct position determination algorithm of heterogeneous sensors based on delay and AOA is derived. Whenever observed signals are narrowband, or SNR is in low level, the proposed algorithm provides much better performance in accuracy than the conventional algorithm. It doesn't matter for localization algorithm about how many numbers of targets and receivers and which type of the transmitter's signal envelope.

\section{REFERENCES}

[1] Ismail Guvenc, Chia-Chin chong, "A Survey on TOA Based Wireless Localization and NLOS Techniques,” IEEE Communications Surveys \& Tutorials, vol. 11, no. 3, pp. 107-124, Third Quarter 2009.

[2] Weiss A, "Direct position determination of narrowband radio frequency transmitters,” IEEE Signal Processing Letters, vol. 11, no. 5, pp. 513516, 2004.

[3] Amar A, Weiss A, "Analysis of direct position determination approach in the presence of model errors,” IEEE, pp. 521-524, 2004.

[4] Oispuu M, "Direct detection and location of multiple sources with intermittent emission”.

[5] Oispuu M, Nickel U, "Direct detection and position determination of multiple sources with intermittent emission," Signal Processing, pp. 3056-3064, 2010.

[6] Kay S, Vankaya N, "Improvement of TDOA position fixing using the likelihood curvature,” IEEE Transactions on Signal Processing, vol. 61, no. 8, pp. 1910-1914, 2013.

[7] Xu Cuichun, Kay S, "On centralized composite detection with distributed sensors,” IEEE, 2008.

[8] Zhiying Huang, "Multi-Array Data Fusion Based Direct Position Determination Algorithm,” IEEE Seventh International Symposium on Computational intelligence and Design, 2014

[9] Xinxin Ouyang, Qun Wan, Jingmin Cao, Jinyu Xiong, Qing He, “Direct TDOA geolocation of multiple frequency-hoppong emitters in flat fading channels,” IET Signal Processing, vol. 11 Iss. 1, pp. 80-85, 2016.

[10] Weiss A, "Direct geolocation of wideband emitters based on delay and Doppler,” IEEE Transactions on Signal Processing, vol. 59, no. 6, 59(6): pp. 2513-2521, 2011.

[11] Amar A, Weiss A, "Advances in direct position determination," IEEE Sensor Array and Multichannel Signal Processing Workshop, pp. 584588, 2004.

[12] Amar A, Weiss A, "Direct position determination in the presence of model errors-known waveforms,” Digital Signal Processing, pp. 584-588, 2006.

[13] Li Jinzhou, Yang le, Guo Fucheng, jiang Wenli, “Coherent summation of multiple short-time signals for direct positioning of a wideband source based on delay and Doppler,” Digital Signal Processing, pp. 59-70, 2016. 